\title{
Balkanologie
}

Balkanologie Revue d'études pluridisciplinaires

Vol. VII, $n^{\circ} 1$ | 2003

Volume VII Numéro 1

\section{Migrations et diasporas : les Balkans mobiles}

\section{Mirjana Morokvašic}

\section{OpenEdition}

\section{Journals}

Édition électronique

URL : http://journals.openedition.org/balkanologie/464

DOI : 10.4000/balkanologie.464

ISSN : 1965-0582

\section{Éditeur}

Association française d'études sur les Balkans (Afebalk)

\section{Édition imprimée}

Date de publication : 1 juin 2003

Pagination : 11-17

ISSN : 1279-7952

\section{Référence électronique}

Mirjana Morokvašic, « Migrations et diasporas : les Balkans mobiles », Balkanologie [En ligne], Vol. VII, $n^{\circ} 1$ | 2003, mis en ligne le 18 février 2009, consulté le 17 décembre 2020. URL : http://

journals.openedition.org/balkanologie/464 ; DOI : https://doi.org/10.4000/balkanologie.464 


\section{DOSSIER}

\section{MIGRATIONS \\ ET DIASPORAS}




\title{
MIGRATIONS ET DIASPORAS : LES BALKANS MOBILES
}

\author{
Mirjana Morokvašić*
}

Avec la chute du monde communiste, le paysage migratoire a profondément changé en Europe. Lorsque celle-ci était encore divisée par le rideau de fer, une double dynamique migratoire avait vu le jour : à l'Est, une mobilité réduite essentiellement à l'espace des pays du COMECON, avec, toutefois, des ouvertures vers l'Occident qui variaient d'un pays à l'autre et dont ne bénéficiait pas l'ensemble des populations de ces pays ; à l'Ouest, la mise en place de la "préférence communautaire"1, le recours à la main d'œuvre immigrée originaire du Sud et l'ouverture aux demandeurs d'asile, en particulier ceux en provenance des pays communistes.

La fracture européenne avait des traits spécifiques dans les Balkans. La Grèce et la Yougoslavie participaient au système de travail migrant européen dans les années 1960 et 1970 en fournissant plusieurs centaines de milliers et environ un million de "Gastarbeiter" respectivement. Après son accession à la Communauté Européenne, la Grèce connaît une forte migration de retour et devient progressivement un pays d'immigration à son tour, tandis que les migrations de Yougoslavie continuent, même après la suspension des recrutements officiels en 1973, sous forme de regroupements familiaux ou de va-etvient (les ressortissants yougoslaves pouvaient circuler librement, sans visa). L'Albanie était fermée ; quant à la Roumanie, seules les migrations ethniques et certaines migrations de travail dans le cadre du COMECON et quelques autres pays ont été possibles. Avec ses frontières ouvertes et sa société de consommation la Yougoslavie fut pendant un certain temps "l'Ouest de l'Est" et les Roumains venaient $y$ travailler ou faire du commerce.

\footnotetext{
- Directrice de recherches au LASP (Laboratoire d'analyse des systèmes politiques), CNRS et Université Paris X. (mirjana.morokvasic@u-paris1o.fr)

1 La libre circulation pour les ressortissants des pays membres de la Communauté Européenne.
} 
La nouvelle donne migratoire après 1989 résulte pour une part des recompositions politiques qui se sont produites à l'Est dans des contextes parfois violents. Des migrations de travail se développent également, elles sont de plus en plus souvent de très courte durée, pendulaires et de proximité, régulières ou non. Dans la grande diversité des phénomènes migratoires déclenchés par ou directement liés au lever du rideau de fer, on peut distinguer des phénomènes anciens qui s'amplifient (demandeurs d'asile) ou se transforment (le système de travail migrant), des phénomènes qui sont ou paraissent nouveaux à l'Ouest mais qui existaient à l'Est (des "touristes", des migrations marchandes, des migrations pendulaires) ; des mouvements "ethniques" (rapatriements ou de "retour") qui ont toujours existé, mais dont l'ampleur et la concentration dans le temps sont exceptionnelles immédiatement après 1989.

Le bouleversement des tendances à long terme à la fin des années 1980 et au début des années 1990 est particulièrement marqué dans les Balkans. L'Albanie sort d'une très longue période d'autarcie. Les départs de ses ressortissants vers l'Italie, fortement médiatisés, nourrissent les scénarios catastrophe sur l'invasion de l'Occident par des "pauvres de l'Est". Ils partent également en Grèce, ensuite dans d'autres pays européens et aux États Unis. Les flux à partir de la Roumanie se diversifient, mais les Roumains attendront 2002 pour pouvoir voyager sans visa dans l'Union européenne. La Grèce a, entre temps, le taux d'immigration le plus élevé d'Europe (près de $10 \%$ ), elle a passé des accords avec l'Albanie et la Bulgarie et a procédé à des régularisations afin d'éponger une partie de la migration irrégulière, notamment en provenance d'Albanie.

Les territoires yougoslaves, parmi les principaux fournisseurs de main d'œuvre à l'Europe de l'Ouest, se sont transformés au début des années 1990 en principale source de réfugiés : trois millions de personnes environ ont fui la guerre ou ont été déplacés de force ; on estime qu'un million d'entre eux se trouve toujours à l'étranger, dont un grand nombre de jeunes diplômés et des élites professionnelles. Si certains ont pu rentrer chez eux, comme les Albanais de Kosovo immédiatement après les bombardements de l'OTAN en $1999^{2}$, la plupart resteront là où ils ont trouvé refuge, c'est-à-dire dans les territoires où ils sont ethniquement majoritaires. Les restes balkaniques de ce que Hannah Arendt appelait "la ceinture des populations mixtes en Europe centrale et orientale" se sont démêlés pour de bon, le "surplus ethnique" a été expulsé ou est parti "volontairement" rejoindre sa majorité dans un nouvel État ou une nouvelle entité territoriale. Par ailleurs, les guerres et les sanctions internationales ont fait fleurir l'économie parallèle, le développement de la migration 
marchande et des trafics en tout genre dans l'ensemble des Balkans 3 . L'analyse de l'ensemble de ces mouvements qui ont précédé, accompagné ou qui ont été la conséquence des guerres yougoslaves reste à faire.

Les cinq textes réunis dans le dossier Migrations et diasporas ont été présentés aux premières rencontres organisées par l'AFEBALK les 19 et 20 décembre 2002 à Paris. Leurs auteurs sont de jeunes chercheurs, doctorants et postdoctorants, qui éclairent plusieurs facettes du paysage migratoire recomposé en Europe en posant leur regard sur les Balkans, et plus particulièrement sur les migrations roumaine et albanaise : le phénomène de circulation (qu'elle soit sporadique ou, par contre, une véritable "installation dans la mobilité"), les conditions d'émergence de diaspora et "la diasporisation accélérée", la migration ethnique et la migration de travail, enfin, la migration transfrontalière.

Qu'il s'agisse de la diaspora albanaise aux États-Unis (Nadège Ragaru et Amilda Dymi) ou celle des Roumains hautement qualifiés au Canada (Michaela Nedelcu), de la migration des Albanais en Grèce (Pierre Sintès), des Roumains en Espagne (Swanie Potot) ou de la circulation des Saxons entre la Roumanie et l'Allemagne (Bénédicte Michalon), le dénominateur commun est une nouvelle dynamique où les réseaux et la mobilité jouent un très grand rôle dans les stratégies élaborées par des acteurs. Les migrants s'appuient sur des réseaux pour construire leur champ migratoire englobant deux ou plusieurs pays où la mobilité et un certain "savoir circuler" sont leur principale ressource.

Il arrive que dans ces stratégies individuelles - qui peuvent devenir un bien collectif ultérieurement, partagé par les membres du réseau - les migrants contournent et/ou instrumentalisent les logiques des États comme le font par exemple les Saxons ou encore les Roumains en Andalousie. La recherche d'un statut stable (l'admission en Allemagne pour les premiers, la régularisation en Espagne pour les seconds) n'est pas motivée par le projet d'installation durable, mais par le projet de libre circulation et par la recherche, par ce biais, des meilleures conditions d'accueil et d'emploi. Les réseaux établis par les uns peuvent aussi servir aux autres, surtout aux membres de la même communauté, mais aussi au-delà des cloisonnements ethniques. Ils peuvent, comme dans l'espace transfrontalier épirote, être ravivés, même après une longue coupure de plusieurs décennies.

Enfin, en dépit de la coupure de la fin des années 1980, les recherches présentées ici soulignent l'importance de la continuité dans l'expérience migratoire : les migrants cherchent à inscrire leurs pratiques dans le prolongement des migrations antérieures, celles datant de la période communiste ou celles d'avant. 
Bénédicte Michalon montre comment la catégorisation ethnique des Saxons (ces "Allemands de Roumanie" qui ont adopté relativement récemment la germanité dans leur identification collective) repose sur un mode de pensée binaire et sur l'opposition migration temporaire - migration définitive. Celleci cloisonne les acteurs dans une logique de retour vers "la mère patrie" et a, jusqu'à présent, écarté de l'observation leurs autres pratiques migratoires. Dans les faits cependant, l'émigration définitive, de retour, est à l'origine de formes de mobilités diversifiées et notamment de la circulation. On peut donc observer une continuité entre l'installation antérieure des Saxons en Allemagne et leur circulation actuelle. Pour opérer le glissement entre l'émigration définitive, qui fut, d'ailleurs, pour les Saxons, la seule possible sous le régime communiste de Ceausescu, et la circulation, ceux-ci mobilisent l'ethnicité en s'appuyant sur des réseaux préexistants : "L'encadrement législatif de l'émigration définitive est employé de manière inédite par les Saxons, demeurés en Roumanie mais candidats à de cours séjours outre-Rhin (...). En ce sens, la circulation saxonne reste une migration ethnique".

Motivées par des raisons familiales à l'origine, ces migrations sporadiques - on n'observe pas une "installation dans la mobilité" comme dans d'autres migrations post-communistes 4 - se transforment en migrations de main d'œuvre. À ce propos, l'auteur signale un autre glissement (possible) : si la mobilité des Saxons en Allemagne reste certes confinée aux structures réticulaires saxonnes, en Roumanie, elle favorise en revanche la migration économique : des "non Saxons" (Roumains, Tsiganes, Magyars) sont progressivement incorporés dans une mobilité vers l'Allemagne et ailleurs dans l'espace Schengen.

C'est ainsi que le savoir et la compétence acquis pour faciliter la circulation sont utilisés par les autres également, à l'instar de ces Roumains adoptant les stratégies chères aux Tsiganes, sans qu'il y ait forcément d'entraide directe entre les groupes. 5 .

Les circulations des Roumains entre Téléorman, une région agricole en crise, et l'Andalousie, en quête de main d'œuvre flexible et bon marché, se situent également dans la continuité des migrations antérieures. En effet, comme l'écrit Swanie Potot, contrairement à d'autres régions roumaines où la population n'a découvert la migration internationale qu'avec la chute du communisme, à Téléorman, les allées-venues vers l'Espagne se situent dans le pro-

4 Voir en particulier : Potot (Swanie), "Les migrants transnationaux : une nouvelle figure sociale en Roumanie", Revue d'Études Comparatives Est-Ouest, 33 (1), mars 2002 ; Diminescu (Dana), Lagrave (Rosemarie), "Faire une saison. Pour une anthropologie des migrations roumaines", Migrations Etudes, (91), novembre-décembre ; Morokvašić (Mirjana), "Une migration pendulaire : les Polonais en Allemagne", Hommes et Migrations, (1155), juin 1992 ; Morokvašić (Mirjana), "La mobilité transnationale comme ressource", Cultures et Conflits, (33-34), 1999.

5 Potot (Swanie), art.cit., p.162. 
longement des pratiques migratoires, notamment celles dans le cadre des accords d'exportation de main d'oeuvre conclus sous Ceausescu entre la Roumanie et certains pays du bloc soviétique et, plus tard, avec la RFA, la Libye, l'Egypte et l'Irak. Se mettre en route pour pallier les difficultés sur place, n'est donc pas nouveau pour la population de cette région, mais fait partie du mode de vie où "chaque famille a l'habitude de voir partir, à intervalle régulier, plusieurs de ses membres durant quelques mois". Ceux qui s'installent dans la mobilité sont généralement les mêmes qui ont déjà eu l'habitude de migrer au temps de la dictature, qui ont acquis un "savoir circuler". Surqualifiés, adaptables, peu revendicatifs et peu visibles, ils ont un profil avantageux et sont appréciés en Andalousie, région qui ne cherche pas à faire état de son besoin de travailleurs immigrés.

Pierre Sintès s'intéresse au rôle des réseaux transfrontaliers dans la configuration et la dispersion de la migration albanaise en Grèce. On peut faire remonter le potentiel de solidarités à la période d'avant la fermeture de l'Albanie, quand la région était un lieu d'échanges intenses. Malgré la séparation par la frontière et le développement disjoint qu'ont connu les populations de part et d'autre de la frontière, les réseaux préexistants sont ravivés : les hommes - souvent âgés, partant en éclaireurs - empruntent les chemins pris par leurs prédécesseurs il y a une cinquantaine d'année.

La plupart de travaux sur les migrations roumaines d'après 1989 portent sur les mobilités irrégulières, la circulation de travailleurs qui sont parfois, certes très qualifiés, mais qui ne trouvent que des emplois précaires et pour qui la migration est une réponse à la crise au pays, une stratégie de survie.

Mihaela Nedelcu innove en observant une migration différente. Les Roumains très qualifiés au Canada sont capables de mobiliser un capital social et culturel dès le départ : "Ils ont des compétences facilement négociables sur un marché global toujours à la recherche des talents et des spécialistes". Ils se mettent en réseau surtout grâce aux nouvelles technologies d'information et de communication, dont ils sont aussi les spécialistes. Non seulement leur profil les distingue de la majorité des migrants actuels de Roumanie, mais leur migration est, selon Nedelcu, de longue durée. Peut-on parler d'une nouvelle diaspora alors que cette migration est relativement récente et comment cette diaspora est-elle organisée ? D'après l'auteur, les jeunes professionnels qu'elle "netnographie", forts de leurs compétences particulières, notamment dans le domaine de la technologie de l'information, utilisent Internet pour mettre en commun leurs expériences migratoires et pour constituer un "collectif circulatoire" 6 qui fédère les "savoir circuler" par l'effet d'émergence d'une mémoire 
collective de la migration de date récente. En quelque sorte, les nouvelles technologies d'information et de communication, sous certaines conditions, seraient susceptibles d'accélérer la diasporisation des communautés roumaines. Celles-ci entretiennent un nouveau rapport au temps et à l'espace : elles sont plus tournées vers le présent et vers l'avenir que vers le passé et, si Internet joue un rôle d'unificateur de ces populations en dispersion, en les mettant en réseau, il contribue cependant à déterritorialiser leurs pratiques.

Sur un autre registre, Nadège Ragaru et Amilda Dymi s'intéressent, elles aussi, à un sujet peu étudié et qui a trait aux diasporas : elles observent les recompositions identitaires chez les Albanais des États-Unis mettant en lumière les conditions d'émergence de la diaspora ainsi que son impact dans les prises de positions des États Unis au moment de la crise au Kosovo en 1998-1999. Les auteurs montrent notamment comment les Albanais des États-Unis, peu unis autour d'un projet national, avec des allégeances souvent "régionales" et "micro-communautaires", trouvent, dans la cause du Kosovo, une force mobilisatrice et unificatrice. Leur influence sur la gestion de la crise, efficace certes, est à mesurer en fonction du contexte américain qui, comme le montrent les auteurs, leur a été particulièrement favorable à une période précise sous l'administration Clinton. À la question sous-jacente à cette analyse, à savoir si la ré-appropriation de l'identité nationale, même si elle passe par une phase de radicalisation ethno-nationaliste, peut être "une ressource au service des projets politiques et sociaux divers" (je rajouterais : autres que le "long distance nationalism"7 belligène), les auteurs répondent par l'affirmative. On est tenté de leur donner raison ; toutefois, il ne faut pas oublier qu'un potentiel du nationalisme radical reste toujours mobilisable quelle que soit la communauté en question.

Les auteurs de ce dossier contribuent à renouveler les interrogations sur les pratiques et les expériences migratoires dans le débat actuel sur les migrations transnationales post-communistes qui a d'ailleurs, souvent, contourné les Balkans. Ils insistent sur la complexité des phénomènes observés et mettent en lumière les pratiques occultées dans la production de connaissance sur les migrations, souvent prisonnière des catégorisations existantes. Ils posent la question du sens même de la migration et du retour dans un contexte mondialisé où les schèmes migratoires classiques sont brouillés et les distances banalisées suite aux progrès des transports et des communications. La migration peut ainsi devenir une alternative à l'émigration, une stratégie pour ne pas

7 Sur le "long distance nationalism", voir Glick-Schiller (Nina), Fouron (Georges E.), Georges Woke up Laughing. Long-Distance Nationalism and the Search for Home, Durham / London : Duke University Press, 2001 ; Anderson (Benedict), Imagined Communities, London : Verso, 1983. 
quitter son pays pour toujours. Le retour, quant à lui, lorsque le départ luimême n'est pas définitif, comme ce fut impérativement le cas pour les exilés du communisme, n'a également plus le même sens qu'auparavant : étant possible à tout moment, donc comme le départ, il n'est pas envisagé de manière définitive, mais se concrétise dans la mobilité, dans des va-et-vient physiques ou, comme on le verra, virtuels.

Sortant des sentiers balisés des études sur la migration et l'intégration, les auteurs, chacun dans son domaine, nous présentent un travail pionnier : ils ont choisi de s'intéresser aux groupes de migrants peu connus, peu étudiés et de taille relativement faible. Ces groupes cependant, à leur tour pionniers et éclaireurs, relient les Balkans à un ailleurs qui leur fut pendant longtemps inaccessible et contribuent ainsi à un processus de normalisation entre des pays ayant eu des échanges réduits pendant la période communiste. Se faisant ils ont un rôle intégrateur et sont au cœur des transformations profondes tant au niveau local et régional qu'au niveau européen et global. 